\title{
Systemic therapy without radiation may be appropriate as neoadjuvant therapy for localized pancreas cancer
}

\author{
Scott Kizy ${ }^{1 \#}$, Ariella M. Altman ${ }^{1 \#}$, Keith M. Wirth ${ }^{1}$, Schelomo Marmor ${ }^{1}$, Jane Y. C. Hui ${ }^{1}$, Todd M. Tuttle ${ }^{1}$, \\ Emil Lou ${ }^{2}$, Khalid Amin ${ }^{3}$, Jason W. Denbo ${ }^{1}$, Eric H. Jensen ${ }^{1}$ \\ ${ }^{1}$ Division of Surgical Oncology, Department of Surgery, ${ }^{2}$ Division of Hematology and Oncology, Department of Internal Medicine, ${ }^{3}$ Department of \\ Laboratory Medicine and Pathology, University of Minnesota, Minneapolis, MN, USA \\ Contributions: (I) Conception and design: S Kizy, AM Altman, S Marmor, EH Jensen; (II) Administrative support: S Kizy, AM Altman, KM Wirth; \\ (III) Provision of study materials or patients: S Kizy, AM Altman, EH Jensen; (IV) Collection and assembly of data: S Kizy, AM Altman, K Amin; \\ (V) Data analysis and interpretation: S Kizy, AM Altman, KM Wirth, S Marmor, K Amin, JW Denbo, EH Jensen; (VI) Manuscript writing: All \\ authors; (VII) Final approval of manuscript: All authors. \\ \#These authors contributed equally to this work. \\ Correspondence to: Eric H. Jensen, MD. Division of Surgical Oncology, Department of Surgery, University of Minnesota, 420 Delaware Street SE, \\ MMC 195, Minneapolis, MN 55455, USA. Email: jense893@umn.edu.
}

\begin{abstract}
Background: The utility of neoadjuvant treatment for resectable pancreas cancer is yet to be determined, but has commonly included chemoradiation. We evaluated outcomes in patients with radiographically resectable pancreatic adenocarcinoma treated with neoadjuvant chemotherapy without chemoradiation.

Methods: A retrospective review of patients in our institutional pancreatic cancer registry was performed, which identified 36 patients who received neoadjuvant chemotherapy alone for resectable pancreatic adenocarcinoma between 2012 and 2016.

Results: Median age at diagnosis was 66.3 years. Chemotherapy regimens included gemcitabine $(\mathrm{n}=17)$, gemcitabine/nab-paclitaxel $(\mathrm{n}=8)$, or 5 -FU/leucovorin/irinotecan/oxaliplatin (FOLFIRINOX) $(n=11)$. Surgical resection was performed in $69 \%$ of patients $(n=25)$, with an $R 0$ resection rate of $92 \%$ ( $n=23$ patients). During chemotherapy, distant disease became apparent in $19 \%$ of patients $(n=7)$, while no patients had evidence of local progression. Resection rates were similar between chemotherapy regimens (single agent $=59 \%$, multiple agent $=79 \%$ ). Median overall survival for all patients who received neoadjuvant chemotherapy was 30.3 and 34.4 months for those who underwent surgical resection. There was no difference in median survival for patients treated with gemcitabine (31.3 months) or multi-agent chemotherapy (29.7 months). Conclusions: A short course of neoadjuvant chemotherapy without chemoradiation may improve patient selection prior to surgical resection for pancreas cancer. Further, local disease progression did not limit surgical resection in this small series.
\end{abstract}

Keywords: Pancreatic adenocarcinoma; chemotherapy; survival; neoadjuvant chemotherapy; resection

Submitted Mar 06, 2019. Accepted for publication Apr 23, 2019.

doi: 10.21037/hbsn.2019.04.17

View this article at: http://dx.doi.org/10.21037/hbsn.2019.04.17

\section{Introduction}

Pancreatic adenocarcinoma is an aggressive malignancy with increasing incidence. In the United States alone there will be an estimated 55,440 new cases of pancreas cancer diagnosed in 2018, and an estimated 44,330 patients will die of the disease in the same year (1). Despite slight improvements in survival, the 5 -year survival for patients with localized disease remains less than $35 \%$ (1).

In patients with resectable disease who undergo upfront surgical resection, randomized controlled trials have shown that the use of adjuvant chemotherapy improves survival $(2,3)$ 
and is recommended by National Comprehensive Cancer Network (NCCN) guidelines (4). However, the proportion of patients who receive recommended chemotherapy after surgical resection is low. Previous studies have shown that at best, only around $60 \%$ of patients complete recommended adjuvant therapy (5-7). A review of the California Cancer Registry found that only $28 \%$ of patients received NCCN guideline compliant care consisting of surgery and chemotherapy or chemoradiation for resectable tumors (7).

As an alternative to the surgery-first approach, neoadjuvant therapy has many theoretical benefits. First, it ensures all patients start systemic therapy which is critical to obtain optimal survival outcomes. Additionally, it allows early aggressive disease to manifest itself, therefore minimizing the chance a patient undergoes surgery only to have disease progression shortly thereafter. Prior studies have shown that neoadjuvant chemotherapy and chemoradiation in resectable pancreatic adenocarcinoma is feasible, however it is unclear if resection rates or overall survival are significantly improved (8-12). Finally, patients who receive upfront chemotherapy are less likely to have positive lymph nodes or positive margins at the time of surgery $(13,14)$. Typically, chemoradiation is used in the neoadjuvant setting in an attempt to optimize local tumor control; however, this therapy may add additional toxicities to the patient.

The aim of the current study was to evaluate a short course of neoadjuvant-intent chemotherapy without radiation in patients diagnosed with radiographically resectable pancreatic adenocarcinoma. Our intent was to determine whether up-front chemotherapy alone led to increased risk of local disease progression, and to assess whether multi-agent chemotherapy regimens were superior to single-agent chemotherapy in the neoadjuvant setting.

\section{Methods}

After obtaining approval from the Institutional Review Board, our institutional Pancreatic Cancer Registry was queried for patients who received neoadjuvant-intent chemotherapy for pancreatic cancer. The Pancreatic Cancer Registry is a retrospective registry of patients who received care for pancreatic adenocarcinoma at the University of Minnesota from 2012-2016 which includes patient demographic, tumor characteristics and outcomes, to be used for institutional research. During this time period, it was institutional practice to treat patients with resectable tumors with neoadjuvant-intent chemotherapy at diagnosis. A short-course of neoadjuvant-intent chemotherapy consisted of two to three months of upfront chemotherapy followed by surgical resection then the completion of the intended chemotherapy course after recovery. At the time of diagnosis, all patients were reviewed in a multi-disciplinary conference to determine appropriate neoadjuvant regimen, weighing patient risk factors and comorbidities. Patients were included in the study if they were at least 18 years old, had biopsy proven adenocarcinoma, radiographically resectable disease and were started on neoadjuvant chemotherapy without radiation.

In order to verify resectability status, imaging at diagnosis was reviewed by two independent and blinded surgical oncologists to confirm the patient had resectable disease at presentation as defined by the NCCN guidelines (lack of arterial involvement and $<180$-degree involvement of superior mesenteric or portal vein) (4). If any disagreements occurred, the cases and imaging were reviewed together until a consensus decision was reached. Patients who did not have radiographically resectable disease at diagnosis were excluded. Patients were excluded if they had borderline resectable tumors, metastatic disease at diagnosis, or were treated with upfront surgical resection. Data for patients who met inclusion criteria were abstracted from our institutional Pancreatic Cancer Registry, which included demographics, pre-treatment evaluation, preoperative $\mathrm{AJCC} 7^{\text {th }}$ edition TNM stage, preoperative imaging, CA19-9 levels, treatment plan, operative reports, pathology, post-chemotherapy and postoperative imaging, adjuvant treatment, complications, disease recurrence, and survival. Post-treatment imaging was re-reviewed by two independent and blinded surgical oncologists in order to assess for radiographic disease progression or regression. Pathologic specimens were reviewed by a blinded pathologist and specimens were given an Evans criteria score (15). The Evans score is a histologic grading scheme to quantify the presence or absence of a response to treatment in pathologic specimens (15). A grade I score indicates characteristic cytologic changes of malignancy but $<10 \%$ tumor destruction, a grade II score indicates $10-90 \%$ of tumor cells destroyed, a grade III score indicates $<10 \%$ of tumor cells are viable and a grade IV score indicates a complete response to treatment with no viable tumor cells present (15). For patients lost to follow-up, an online obituary search was performed as an attempt to determine vital status and date of death.

Patients were analyzed with an intention-to-treat protocol and grouped by neoadjuvant chemotherapy regimen: gemcitabine alone versus multi-agent chemotherapy (gemcitabine/nab-paclitaxel or FOLFIRINOX). Patient and 
Table 1 Patient and tumor characteristics at diagnosis for all patients with resectable pancreatic adenocarcinoma treated with neoadjuvant chemotherapy $(\mathrm{n}=36)$

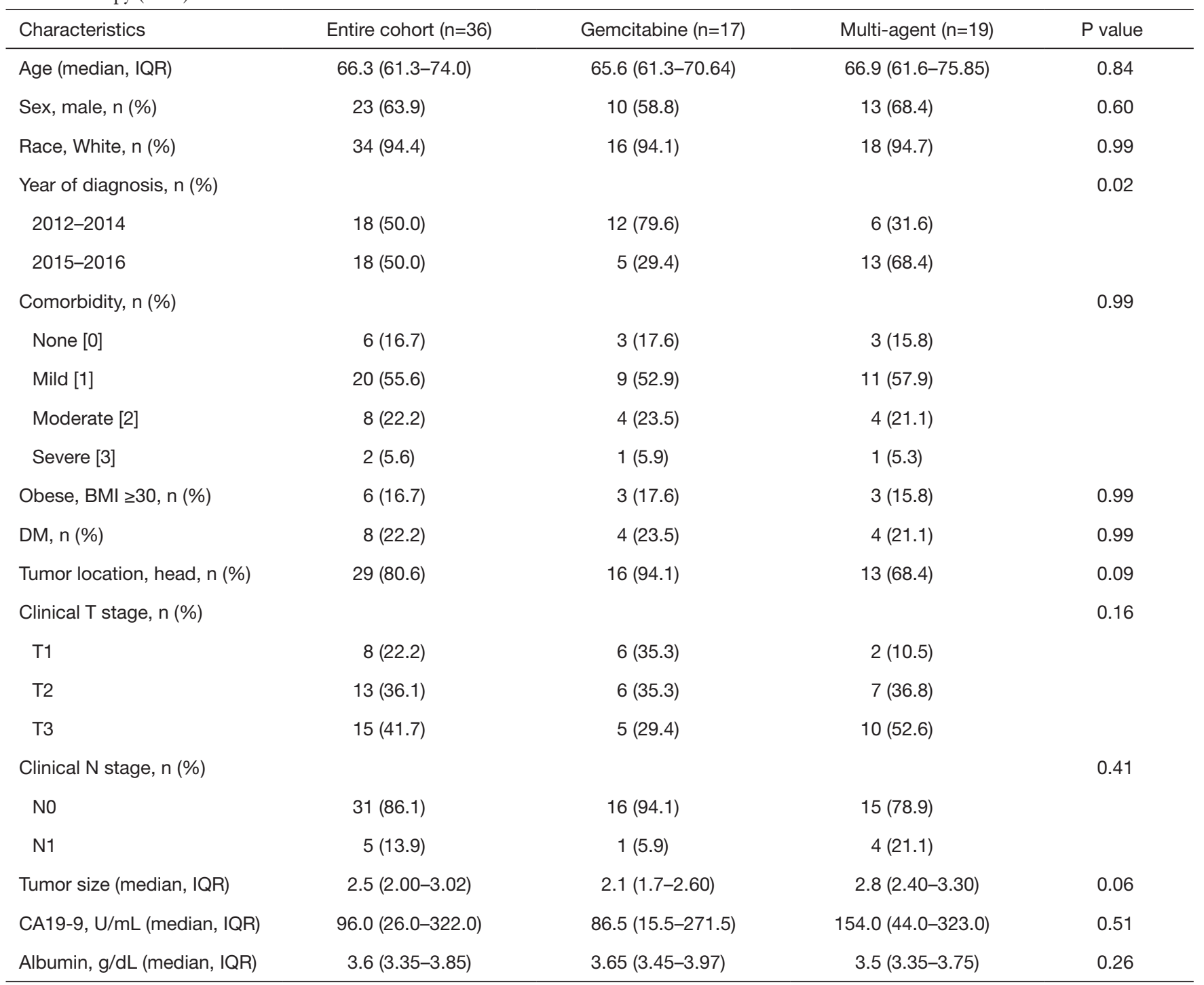

BMI, body mass index; DM, diabetes mellitus.

tumor characteristics were evaluated utilizing a two-tailed Fisher's exact test for categorical variables, Student's $t$-test for continuous variables and Kruskal-Wallis rank sum test for non-parametric continuous variables. Survival was evaluated utilizing the Kaplan Meier method and log rank test. Statistical significance was defined as a $P$ value of $\leq 0.05$. Statistical analyses were performed utilizing $\mathrm{R}(\mathrm{R}$ version 3.4.2) and Prism (7.00, GraphPad Software, La Jolla, CA, USA).

\section{Results}

\section{Patient and tumor characteristics}

A total of 36 patients with resectable pancreatic adenocarcinoma who met inclusion criteria were identified (Table 1). The median age was 66.3 years [interquartile range (IQR), 61.3-74.0], 64\% ( $\mathrm{n}=23)$ were male, $94 \%(\mathrm{n}=34)$ were white, $17 \%(\mathrm{n}=6)$ of patients were obese (BMI $\geq 30)$, and $22 \%$ 
Table 2 Treatment characteristics for patients treated with neoadjuvant chemotherapy

\begin{tabular}{|c|c|c|c|c|}
\hline Variables & Entire cohort $(n=36)$ & Gemcitabine $(n=17)$ & Multi-agent $(n=19)$ & $P$ value \\
\hline Gemcitabine & $17(47.2)$ & $17(100.0)$ & $0(0.0)$ & \\
\hline Gem/nab-paclitaxel & $8(22.2)$ & $0(0.0)$ & $8(42.1)$ & \\
\hline FOLFIRINOX & $11(30.6)$ & $0(0.0)$ & $11(57.9)$ & \\
\hline Surgical decision, $\mathrm{n}(\%)$ & & & & 0.48 \\
\hline Proceed to surgery & $29(80.6)$ & $13(76.5)$ & $16(84.2)$ & \\
\hline Distant progression & $3(8.3)$ & $2(11.8)$ & $1(5.3)$ & \\
\hline Local progression & $0(0.0)$ & $0(0.0)$ & $0(0.0)$ & \\
\hline
\end{tabular}

$(n=8)$ had a diagnosis of type-2 diabetes mellitus. Eighty percent $(n=29)$ of patients had tumors located in the head of the pancreas, and $22 \%$ were $\mathrm{T}$ stage $\mathrm{T} 1,36 \% \mathrm{~T} 2$, and $42 \%$ T3. Median CA19-9 at diagnosis was $96 \mathrm{U} / \mathrm{mL}$ (IQR, 26-322 U/mL) and median albumin was $3.6 \mathrm{~g} / \mathrm{dL}$ (IQR, 3.35-3.85 g/dL). Patients treated with multi-agent chemotherapy had slightly larger tumors (median 2.80 versus $2.10, \mathrm{P}=0.06$ ) and were more likely to be diagnosed in 2015-2016 versus 2012-2014 ( $\mathrm{P}=0.02)$.

\section{Neoadjuvant treatment}

Of the 36 patients eligible for the study, $47 \%(n=17)$ started treatment with gemcitabine alone and $53 \%(\mathrm{n}=19)$ were treated with multi-agent chemotherapy (gemcitabine/nabpaclitaxel or FOLFIRINOX). Among those 19 patients, 8 patients were treated with gemcitabine/nab-paclitaxel and 11 patients with FOLFIRINOX. Twelve percent of patients $(n=2)$ who began treatment with gemcitabine alone were switched to a multi-agent regimen, both due to the identification of distant metastases within 4 months of starting neoadjuvant-intent chemotherapy. Among patients started on multi-agent neoadjuvantintent chemotherapy, $26 \%(\mathrm{n}=5)$ had a change in treatment regimen; three patients treated with gemcitabine/nabpaclitaxel were switched to gemcitabine alone due to comorbidities and poor tolerability, two patients who started on FOLFIRINOX were switched to gemcitabine/ nab-paclitaxel due to the development of complications.

The median time from diagnosis to initiation of chemotherapy was 21 days (IQR, 17-29 days) for all patients. The median duration of chemotherapy (time from initiation of neoadjuvant-intent chemotherapy to surgery) was 88 days (IQR, 76-105 days). For patients who proceeded to surgery, the median interval from diagnosis to surgery was 113 days (IQR, 104-143 days).

\section{Treatment and surgical outcomes}

Treatment outcomes after neoadjuvant-intent chemotherapy are summarized in Table 2. No patient had evidence of local disease progression on re-staging imaging. However, $8 \%$ of patients $(n=3)$ had distant metastatic disease which became evident during chemotherapy, two of which were on single agent gemcitabine. An additional 11\% ( $n=4)$ had clinical deterioration precluding surgery, two patients were on single-agent chemotherapy and two patients were on multiagent.

The median change in CA19-9 levels for all patients while on neoadjuvant-intent chemotherapy was a decrease by $8 \mathrm{U} / \mathrm{mL}$ (IQR, -55 to $0 \mathrm{U} / \mathrm{mL}$ ). The median change in CA19-9 for those treated with single agent chemotherapy was a decrease by $9 \mathrm{U} / \mathrm{mL}$ (IQR, -46.5 to $0.5 \mathrm{U} / \mathrm{mL}$ ); while the median change for those treated with a multiagent regimen was a decrease by $2 \mathrm{U} / \mathrm{mL}$ (IQR, -63.5 to $0 \mathrm{U} / \mathrm{mL}$ ). Among patients who experienced disease progression, the median CA19-9 at diagnosis was $95 \mathrm{U} / \mathrm{mL}$ (IQR, 56.5-301.5 U/mL) and the median change in CA199 was $-8 \mathrm{U} / \mathrm{mL}$ (IQR, -84 to $-5 \mathrm{U} / \mathrm{mL}$ ). For patients who proceeded to surgical resection, the median CA19-9 at diagnosis was $96 \mathrm{U} / \mathrm{mL}$ (IQR, 12.5 to $318 \mathrm{U} / \mathrm{mL}$ ) and the median change in CA19-9 with treatment was $-10 \mathrm{U} / \mathrm{mL}$ (IQR, 
Table 3 Surgical outcomes among the patients who underwent surgical resection $(\mathrm{n}=25)$

\begin{tabular}{lccc}
\hline Variables & Entire cohort, $\mathrm{n}(\%)$ & Gemcitabine, $\mathrm{n}(\%)$ & Multi-agent chemotherapy, $\mathrm{n}(\%)$ \\
\hline Surgical procedure & $20(80.0)$ & $9(90.0)$ & $11(73.3)$ \\
Pancreaticoduodenectomy value & $1(10.0)$ & $4(26.7)$ \\
Distal pancreatectomy & $5(20.0)$ & & 0.61 \\
Surgical outcomes & & $1(10.0)$ & $13.3)$ \\
Vascular resection & $3(12.0)$ & $9(90.0)$ & $14(93.3)$ \\
Margin status, Ro & $23(92.0)$ & & $11(73.3)$ \\
Pathologic outcomes & & $5(50.0)$ & 1.00 \\
Nodal metastases & $16(64.0)$ & & $7(46.7)$ \\
Evans grade & & $4(40.0)$ & $7(46.7)$ \\
I & $11(44.0)$ & $5(50.0)$ & $1(6.7)$ \\
II & $12(48.0)$ & $0(0.0)$ & 0.44 \\
III & $1(4.0)$ & $1(10.0)$ & 0.52 \\
IV & $1(4.0)$ & $(0.0)$
\end{tabular}

a, Evans grade: based on Evans criteria where grade I represents characteristic cytologic changes of malignancy with $<10 \%$ tumor destruction; grade II represents cytologic changes of malignancy with $10 \%$ to $90 \%$ of tumor cells destroyed; grade III represents $<10 \%$ viable-appearing tumor cells present; grade IV represents the presence of no viable tumor cells (15).

-55 to $0 \mathrm{U} / \mathrm{mL})$.

Among all patients who were started on neoadjuvant chemotherapy, $81 \%$ of patients $(n=29)$ proceeded to the operating room, and $69 \%$ of patients $(n=25)$ underwent pancreatic resection. Four patients underwent surgical exploration only, without resection, due to the intraoperative discovery of metastatic disease despite no evidence of disease on pre-operative pancreas protocol CT performed within 3 weeks of surgery. There was no statistically significant difference in resection rates between patients who began treatment with gemcitabine alone versus multi-agent chemotherapy $(59 \%$ in the gemcitabine cohort and $79 \%$ in the multi-agent cohort; $\mathrm{P}=0.34$ ).

Surgical and oncologic outcomes for the 25 patients who underwent a surgical resection are included in Table 3. Eighty percent of patients $(n=20)$ had a pancreaticoduodenectomy and $20 \%(\mathrm{n}=5)$ underwent a distal pancreatectomy. The overall $\mathrm{R} 0$ resection rate was $92 \%(\mathrm{n}=23)$. There was no difference in $\mathrm{R} 0$ resection rates based on chemotherapy regimen (90\% with gemcitabine alone, $93 \%$ with multiagent chemotherapy, $\mathrm{P}=1.00)$. Venous resection was required in $12 \%$ of cases $(n=3)$ and the lymph node positivity rate was $64 \%(n=16)$. On pathologic assessment of tumors, 11 patients (44\%) had a pathologic Evans grade I treatment effect, while most patients (12, 48\%) had an Evans grade
II response. There was no difference in the response to chemotherapy between single agent versus multi-agent therapy $(\mathrm{P}=0.524)$. One patient in the single agent arm had a complete pathologic response to chemotherapy.

\section{Overall survival}

Median follow up for the cohort was 24.7 months. The overall median survival was 30.3 months (Figure 1A). On intention to treat analysis, there was no difference in overall survival between patients treated with gemcitabine alone (median survival of 31.3 months) and those treated with multi-agent chemotherapy (median survival of 29.7 months, Figure $1 B ; \mathrm{P}=0.90)$. Overall survival for the 25 patients who underwent surgical resection was 34.4 months, while overall survival for the 11 patients who did not proceed to resection was 19.1 months (Figure $1 C, \mathrm{P}=0.23$ ).

\section{Discussion}

Pancreatic adenocarcinoma is an aggressive and morbid disease. Patients with resectable disease often have the best prognosis, however even after optimal treatment, median survival remains only 22-28 months, with merely $35 \%$ of patients surviving at 5 years $(1,2)$. The proposed 
A

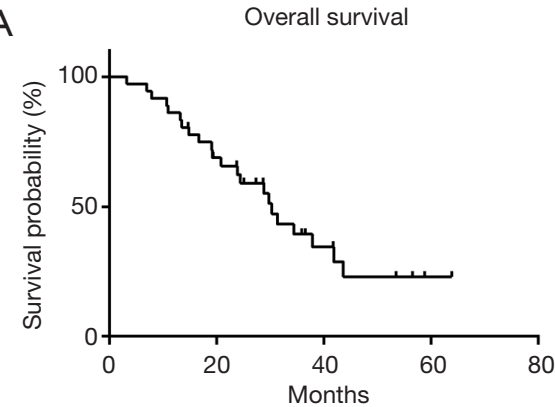

C

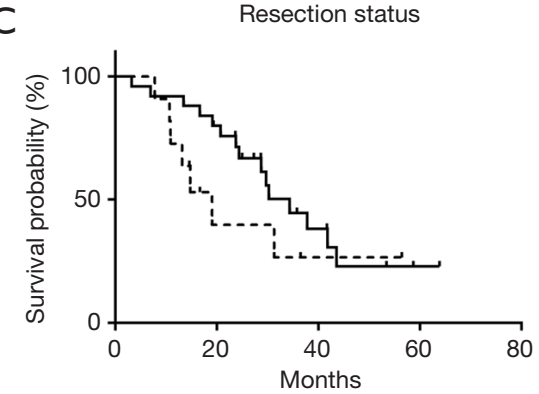

B

- ᄂ. No resection

- Surgical resection

Figure 1 Kaplan-Meier survival curves for patients treated with neoadjuvant-intent chemotherapy. (A) Overall survival for all patients with resectable pancreatic adenocarcinoma initiated on neoadjuvant chemotherapy ( $\mathrm{n}=36$, median survival 30.3 months). (B) Overall survival for all patients with resectable pancreatic adenocarcinoma treated with neoadjuvant chemotherapy, stratified by regimen (gemcitabine alone 31.3 months, vs. multi-agent chemotherapy 29.7 months, $\mathrm{P}=0.90)$. (C) Overall survival for all patients with resectable pancreatic adenocarcinoma treated with neoadjuvant chemotherapy stratified by surgical resection (resection 34.4 months, vs. no resection 19.1 months, $\mathrm{P}=0.23$ ).

benefits of neoadjuvant chemotherapy are that it offers upfront systemic treatment to patients in an attempt to treat micrometastatic disease, assures a greater proportion of patients receive systemic chemotherapy and improves patient selection prior to surgical resection. We report a series of 36 patients who were treated on an intentionto-treat basis with neoadjuvant chemotherapy prior to surgical resection. Under this protocol, there was no local progression of disease, and $69 \%$ of patients proceeded to have surgical resection. Median overall survival for the entire cohort was 30.3 months, and was 34.4 months for patients who proceeded to resection. Although there was a trend towards higher resection rates among patients treated with multi-agent chemotherapy, overall survival was comparable among patients treated with multiagent chemotherapy and those treated with single agent gemcitabine.

In the present study, a short course of neoadjuvantintent chemotherapy was given to patients with resectable disease prior to reimaging and surgical planning. No patients had local progression of disease during neoadjuvant chemotherapy, supporting the use of systemic chemotherapy in the absence of radiation. This technique effectively provided the beneficial systemic effects of chemotherapy without the toxic side-effects of chemoradiation. The limiting factor precluding patients from surgical resection in this series was most commonly the discovery of distant metastatic disease, which was identified in almost $20 \%$ of patients who received neoadjuvant-intent chemotherapy. These patients that developed metastatic disease within 2-3 months of diagnosis and while on chemotherapy, likely had micrometastatic disease at the time of diagnosis. Therefore, this subset of patients would not have benefited from up-front surgical resection, as progression of disease while on systemic treatment is typically indicative of unfavorable tumor biology.

Although most patients had CA19-9 producing tumors, CA19-9 level at diagnosis did not correlate with more aggressive disease. Similarly, reduction of CA19-9 during chemotherapy did not predict resectability in our small cohort.

Although we did not identify a statistically improved survival for patients who received multi-agent therapy, we did identify a few notable trends. Patients who received 
multi-agent therapy seemed to be less likely to develop metastatic disease during chemotherapy despite having similar overall survival compared to the single agent group. While there was no difference in pathologic response to chemotherapy, there was a trend towards higher resection rates in those receiving multi-agent chemotherapy.

Neoadjuvant chemotherapy offers several advantages for patients with pancreatic cancer. Neoadjuvant chemotherapy administered to patients who may harbor undetected systemic metastases may prevent early development of metastatic disease and thus early recurrence after surgery. Furthermore, it has been shown that compliance with recommended treatment guidelines is very poor in pancreatic cancer (7); at least $40 \%$ of patients who undergo surgical resection never receive postoperative systemic therapy $(6,16)$. Therefore, neoadjuvant chemotherapy enables more patients to receive necessary systemic treatment. Almost $30 \%$ of patients experience a serious complication postoperatively, which has been shown to significantly decreases their likelihood of receipt and completion of adjuvant chemotherapy $(6,17)$. Lastly, neoadjuvant chemotherapy allows additional time from diagnosis to surgery to possibly unmask aggressive tumor characteristics of disease which may progress early, potentially sparing these patients from the morbidity of an unnecessary operation.

There are minimal data to guide clinician decision making surrounding neoadjuvant chemotherapy in patients with resectable pancreatic adenocarcinoma. Current NCCN guidelines recommend that patients with resectable disease may be considered for neoadjuvant therapy as part of a study protocol or with high risk features, otherwise they recommend upfront surgical resection (4). The present study supports the use of neoadjuvant chemotherapy alone prior to surgical resection in patients with resectable disease.

While this study is novel in describing a short course of neoadjuvant-intent chemotherapy without radiation in patients with pancreatic adenocarcinoma, we recognize several important limitations. As a retrospective singleinstitution chart review, there is inherent selection bias to the patients who were administrated neoadjuvant chemotherapy, as well as selection of specific chemotherapy agent. However, comparative statistics between the two groups only found a significant difference corresponding to date of diagnosis and not between patient characteristics. Lastly as a retrospective, single-institutional series the sample size of patients treated with neoadjuvant-intent chemotherapy is small; however, it is comparable to the current available studies on neoadjuvant chemotherapy in resectable pancreatic cancer.

Despite its limitations, this study shows that a short course of neoadjuvant-intent chemotherapy for resectable pancreatic adenocarcinoma is safe and potentially effective at selecting patients most likely to benefit from surgical resection. Under this treatment protocol, there was no local progression of disease and $69 \%$ of patients underwent surgical resection with a $92 \%$ R0 resection rate. Amongst those patients who did undergo surgery, median survival was 34.4 months, which compares favorably to patients who undergo upfront surgery followed by adjuvant gemcitabine plus capecitabine (28 months) (2). Survival outcomes in patients treated with neoadjuvant-intent gemcitabine alone was comparable to treatment with multi-agent chemotherapy. While we show that a short course of neoadjuvant-intent chemotherapy in patients with resectable disease is safe and offers good surgical and oncologic outcomes, further large scale, randomized trials are needed to fully characterize the role of neoadjuvant chemotherapy in patients with resectable disease.

\section{Acknowledgments}

The authors of this manuscript would like to thank Stephanie Lundgren and the entire Division of Surgical Oncology at the University of Minnesota for their assistance in the creation of this manuscript. The Abstract was previously presented at the Pancreas Club Annual Meeting, Washington, DC June 2nd, 2018.

Funding: This work was supported by the following funding: Ariella Altman is in part supported by the Institute of Basic and Applied Research in Surgery and the VFW fund of the University of Minnesota. Keith Wirth is supported by National Institutes of Health/National Institute of Diabetes and Digestive Kidney Diseases T32DK108733 (MPI: Yamamoto and Beilman).

\section{Footnote}

Conflicts of Interest: All authors have completed the ICMJE uniform disclosure form (available at https://hbsn. amegroups.com/article/view/10.21037/hbsn.2019.04.17/coif). The authors have no conflicts of interest to declare.

Ethical Statement: The authors are accountable for all aspects of the work in ensuring that questions related 
to the accuracy or integrity of any part of the work are appropriately investigated and resolved. Approval was obtained from the Institutional Review Board (No. 1612E01221). Our institutional Pancreatic Cancer Registry was queried for patients who received neoadjuvant-intent chemotherapy for pancreatic cancer.

Open Access Statement: This is an Open Access article distributed in accordance with the Creative Commons Attribution-NonCommercial-NoDerivs 4.0 International License (CC BY-NC-ND 4.0), which permits the noncommercial replication and distribution of the article with the strict proviso that no changes or edits are made and the original work is properly cited (including links to both the formal publication through the relevant DOI and the license). See: https://creativecommons.org/licenses/by-nc-nd/4.0/.

\section{References}

1. Pancreatic Cancer - Cancer Stat Facts. 2018. Available online: https://seer.cancer.gov/statfacts/html/pancreas.html

2. Neoptolemos JP, Palmer DH, Ghaneh P, et al. Comparison of adjuvant gemcitabine and capecitabine with gemcitabine monotherapy in patients with resected pancreatic cancer (ESPAC-4): a multicentre, open-label, randomised, phase 3 trial. Lancet 2017;389:1011-24.

3. Oettle H, Neuhaus P, Hochhaus A, et al. Adjuvant chemotherapy with gemcitabine and long-term outcomes among patients with resected pancreatic cancer: the CONKO-001 randomized trial. JAMA 2013;310:1473-81.

4. Tempero MA, Malafa MP, Al-Hawary M, et al. Pancreatic Adenocarcinoma, Version 1.2018. Clinical Practice Guidelines in Oncology: National Comprehensive Cancer Network April 27, 2018.

5. Weinrich M, Bochow J, Kutsch AL, et al. High compliance with guideline recommendations but low completion rates of adjuvant chemotherapy in resected pancreatic cancer: A cohort study. Ann Med Surg (Lond) 2018;32:32-7.

6. Merkow RP, Bilimoria KY, Tomlinson JS, et al.

Postoperative complications reduce adjuvant chemotherapy use in resectable pancreatic cancer. Ann Surg 2014;260:372-7.

7. Visser BC, Ma Y, Zak Y, et al. Failure to comply with NCCN guidelines for the management of pancreatic cancer compromises outcomes. HPB (Oxford) 2012;14:539-47.

8. Reni M, Balzano G, Zanon S, et al. Safety and efficacy of preoperative or postoperative chemotherapy for resectable pancreatic adenocarcinoma (PACT-15): a randomised, open-label, phase 2-3 trial. Lancet Gastroenterol Hepatol 2018;3:413-23.

9. Palmer DH, Stocken DD, Hewitt H, et al. A randomized phase 2 trial of neoadjuvant chemotherapy in resectable pancreatic cancer: gemcitabine alone versus gemcitabine combined with cisplatin. Ann Surg Oncol 2007;14:2088-96.

10. O Reilly EM, Perelshteyn A, Jarnagin WR, et al. A single-arm, nonrandomized phase II trial of neoadjuvant gemcitabine and oxaliplatin in patients with resectable pancreas adenocarcinoma. Ann Surg 2014;260:142-8.

11. Tajima H, Ohta T, Kitagawa H, et al. Pilot study of neoadjuvant chemotherapy with gemcitabine and oral S-1 for resectable pancreatic cancer. Exp Ther Med 2012;3:787-92.

12. Heinrich S, Pestalozzi B, Lesurtel M, et al. Adjuvant gemcitabine versus NEOadjuvant gemcitabine/oxaliplatin plus adjuvant gemcitabine in resectable pancreatic cancer: a randomized multicenter phase III study (NEOPAC study). BMC Cancer 2011;11:346.

13. Berriochoa CA, Abdel-Wahab M, Leyrer CM, et al. Neoadjuvant chemoradiation for non-metastatic pancreatic cancer increases margin-negative and node-negative rates at resection. J Dig Dis 2017;18:642-9.

14. Roland CL, Yang AD, Katz MH, et al. Neoadjuvant therapy is associated with a reduced lymph node ratio in patients with potentially resectable pancreatic cancer. Ann Surg Oncol 2015;22:1168-75.

15. Evans DB, Rich TA, Byrd DR, et al. Preoperative chemoradiation and pancreaticoduodenectomy for adenocarcinoma of the pancreas. Arch Surg 1992;127:1335-9.

16. Tzeng CW, Tran Cao HS, Lee JE, et al. Treatment sequencing for resectable pancreatic cancer: influence of early metastases and surgical complications on multimodality therapy completion and survival. J Gastrointest Surg 2014;18:16-24; discussion 24-5.

17. Schwarz L, Bruno M, Parker NH, et al. Active Surveillance for Adverse Events Within 90 Days: The Standard for Reporting Surgical Outcomes After Pancreatectomy. Ann Surg Oncol 2015;22:3522-9.

Cite this article as: Kizy S, Altman AM, Wirth KM, Marmor S, Hui JY, Tuttle TM, Lou E, Amin K, Denbo JW, Jensen EH. Systemic therapy without radiation may be appropriate as neoadjuvant therapy for localized pancreas cancer. HepatoBiliary Surg Nutr 2020;9(3):296-303. doi: 10.21037/ hbsn.2019.04.17 